\title{
ON INNER PARALLEL BODIES AND QUERMASSINTEGRALS
}

\author{
M. A. HERNÁNDEZ CIFRE AND E. SAORÍN
}

\begin{abstract}
Due to the "uncontrollable behavior" of the inner parallel bodies of a convex body $K \subset \mathbb{R}^{n}$ regarding its boundary structure, it is not possible to get precise formulae for their volume/quermassintegrals, contrary to the case of the outer parallel bodies. In this paper we provide (sharp) bounds for the quermassintegrals of the inner parallel bodies, studying previously some particular properties of their boundary in terms of their outer normal vectors.
\end{abstract}

\section{INTRODUCTION}

Let $\mathcal{K}^{n}$ be the set of all convex bodies, i.e., compact convex sets in the $n$-dimensional Euclidean space $\mathbb{R}^{n}$. The subset of $\mathcal{K}^{n}$ consisting of all convex bodies with non-empty interior is denoted by $\mathcal{K}_{0}^{n}$. Let $B_{n}$ be the $n$ dimensional unit ball and $\mathbb{S}^{n-1}$ the $(n-1)$-dimensional unit sphere of $\mathbb{R}^{n}$. The volume of a set $M \subset \mathbb{R}^{n}$, i.e., its $n$-dimensional Lebesgue measure, is denoted by $\mathrm{V}(M)$.

For two convex bodies $K \in \mathcal{K}^{n}$ and $E \in \mathcal{K}_{0}^{n}$ and a non-negative real number $\lambda$ the outer parallel body of $K$ (relative to $E$ ) at distance $\lambda$ is the Minkowski sum $K+\lambda E$. On the other hand, for $0 \leq \lambda \leq \mathrm{r}(K ; E)$ the inner parallel body of $K$ (relative to $E$ ) at distance $\lambda$ is the set

$$
K \sim \lambda E=\left\{x \in \mathbb{R}^{n}: \lambda E+x \subset K\right\},
$$

where the relative inradius $\mathrm{r}(K ; E)$ of $K$ with respect to $E$ is defined by

$$
\mathrm{r}(K ; E)=\sup \left\{r: \exists x \in \mathbb{R}^{n} \text { with } x+r E \subset K\right\} .
$$

When $E=B_{n}, \mathrm{r}\left(K ; B_{n}\right)=\mathrm{r}(K)$ is the classical inradius (see [2, p. 59]). Clearly if $\lambda=0$ the original body $K$ is obtained. Notice that $K \sim \operatorname{r}(K ; E) E$ is the set of (relative) incenters of $K$, usually called kernel of $K$ with respect

2000 Mathematics Subject Classification. Primary 52A20, 52A39; Secondary 52A40.

Key words and phrases. Inner parallel body, quermassintegral, tangential body, extreme vector, form body.

First author is supported by Dirección General de Investigación (MEC) MTM200764504 and by "Programa de Ayudas a Grupos de Excelencia de la Región de Murcia", Fundación Séneca, Agencia de Ciencia y Tecnología de la Región de Murcia (Plan Regional de Ciencia y Tecnología 2007/2010), 04540/GERM/06. Second author is supported by Project Phenomena in High Dimension MRTN-CT-2004-511953 of the European Community. 
to $E$ and denoted by $\operatorname{ker}(K ; E)$. The dimension of $\operatorname{ker}(K ; E)$ is strictly less than $n$ (see [2, p. 59]).

There is an essential geometrically intuitive difference between outer and inner parallel bodies of a convex body $K \in \mathcal{K}^{n}$. On the one hand, the difference lies in the fact that outer parallel bodies are built just by using a vectorial operation in the Euclidean space, while inner parallel bodies do not correspond to any such operation. On the other hand it is precisely this difference what makes the study of inner parallel bodies not only interesting but useful, since it is connected with other nice problems for convex bodies (see, for instance, [1, 4, 7, 8, 10, 11, 14]).

In the case of outer parallel bodies, both the boundary structure and the volume are controlled; there exists an explicit formula for computing the volume of $K+\lambda E$, the so called relative Steiner formula

$$
\mathrm{V}(K+\lambda E)=\sum_{i=0}^{n}\left(\begin{array}{l}
n \\
i
\end{array}\right) \mathrm{W}_{i}(K ; E) \lambda^{i} .
$$

The coefficients $\mathrm{W}_{i}(K ; E)$ are called the relative quermassintegrals of $K$, and they are just a special case of the more general mixed volumes for which we refer to [17, s. 5.1] and [6, s. $6.2,6.3]$. In particular, we have $\mathrm{W}_{0}(K ; E)=$ $\mathrm{V}(K)$ and $\mathrm{W}_{n}(K ; E)=\mathrm{V}(E)$. Analogous formulae to (1.1) give the value of the relative $i$-th quermassintegral of $K+\lambda E$, namely

$$
\mathrm{W}_{i}(K+\lambda E ; E)=\sum_{k=0}^{n-i}\left(\begin{array}{c}
n-i \\
k
\end{array}\right) \mathrm{W}_{i+k}(K ; E) \lambda^{k},
$$

for $\lambda \geq 0$ and $i=0, \ldots, n$. However, the boundary structure of the inner parallel bodies is rather more difficult to control (see Figure 1) and moreover, there is no way to compute (in general) their volume.
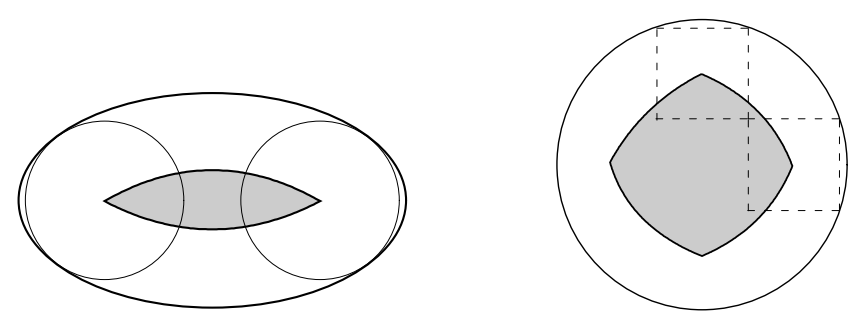

FiguRE 1. Inner parallel body of an ellipse (relative to $B^{2}$ ) and a circle (relative to the square).

It leads us to consider the problem of obtaining lower/upper bounds for the quermassintegrals of the inner parallel body in terms of the magnitudes of the original body, for which it will be crucial to study the behavior of the inner parallel body itself with respect to the structure of its boundary (in the sense we will introduce later). In [1] the case of the volume is studied. 


\section{The MAIN RESUlts}

From now on $E \in \mathcal{K}_{0}^{n}$ will be a fixed convex body (with interior points) and for any $K \in \mathcal{K}^{n}$ we will write $\mathrm{r}=\mathrm{r}(K ; E)$ for the sake of brevity, unless it is not clear from context.

In order to state the main results we need some additional definitions and notation. As usual in the literature, we denote by $h(K, u)=\sup \{\langle x, u\rangle$ : $x \in K\}, u \in \mathbb{R}^{n}$, the support function of $K \in \mathcal{K}^{n}$ (see e.g. [17, s. 1.7]). For convex bodies $K_{1}, \ldots, K_{m} \in \mathcal{K}^{n}$ and real numbers $\lambda_{1}, \ldots, \lambda_{m} \geq 0$, the volume of the linear combination $\lambda_{1} K_{1}+\cdots+\lambda_{m} K_{m}$ is expressed as a polynomial of degree $n$ in the variables $\lambda_{1}, \ldots, \lambda_{m}$,

$$
\mathrm{V}\left(\lambda_{1} K_{1}+\cdots+\lambda_{m} K_{m}\right)=\sum_{i_{1}=1}^{m} \cdots \sum_{i_{n}=1}^{m} \mathrm{~V}\left(K_{i_{1}}, \ldots, K_{i_{n}}\right) \lambda_{i_{1}} \cdots \lambda_{i_{n}} .
$$

The coefficients $\mathrm{V}\left(K_{i_{1}}, \ldots, K_{i_{n}}\right)$ are the mixed volumes of $K_{1}, \ldots, K_{m}$. This formula (mixed volumes) extends the relative Steiner formula (1.1) (relative quermassintegrals). Moreover, if $K_{1}, \ldots, K_{n-1} \in \mathcal{K}^{n}$, the mixed surface area measure $\mathrm{S}\left(K_{1}, \ldots, K_{n-1} ; \cdot\right)$ is the unique finite Borel measure on $\mathbb{S}^{n-1}$ such that for all $K \in \mathcal{K}^{n}$,

$$
\mathrm{V}\left(K, K_{1}, \ldots, K_{n-1}\right)=\frac{1}{n} \int_{\mathbb{S}^{n-1}} h(K, u) d \mathrm{~S}\left(K_{1}, \ldots, K_{n-1} ; u\right) .
$$

For the sake of brevity we will use the abbreviation $\left(K_{1}\left[r_{1}\right], \ldots, K_{m}\left[r_{m}\right]\right) \equiv$ $\left(K_{1}, \stackrel{\left(r_{1}\right)}{\cdot}, K_{1}, \ldots, K_{m}, \stackrel{\left(r_{m}\right)}{\cdot}, K_{m}\right)$. For a deep study of mixed volumes and mixed surface area measures we refer to [17, s. 5.1].

On the other hand, we write $N(K, x)$ to denote the normal cone of $K$ at $x \in \mathrm{bd} K$, i.e., the set of all outer normal vectors of $K$ at $x$ (with the zero vector). A vector $u \in \mathbb{S}^{n-1}$ is an $r$-extreme normal vector of $K, 0 \leq r \leq n-1$, if we cannot write $u=u_{1}+\cdots+u_{r+2}$, with $u_{i}$ linearly independent normal vectors at one and the same boundary point of $K$. We denote the set of $r$-extreme normal vectors of $K$ by $\mathcal{U}_{r}(K)$. Clearly each $r$-extreme normal vector is also an $s$-extreme one for $r<s \leq n-1$. Notice that for $K \in \mathcal{K}^{n}$, if $x \in \mathrm{bd} K$ is a regular point (i.e., if the supporting hyperplane to $K$ at $x$ is unique), then $\operatorname{dim} N(K, x)=1$ and hence the (only) outer normal vector $u \in N(K, x)$ is a 0 -extreme normal vector of $K$. A convex body $K$ is said to be regular if all its boundary points are regular.

The (relative) form body of a convex body $K \in \mathcal{K}_{0}^{n}$ with respect to $E$, denoted by $K^{*}$, is defined as

$$
K^{*}=\bigcap_{u \in \mathcal{U}_{0}(K)}\{x:\langle x, u\rangle \leq h(E, u)\}
$$

A convex body $K \in \mathcal{K}^{n}$ containing the convex body $E \in \mathcal{K}_{0}^{n}$ is called a $p$ tangential body of $E, p \in\{0, \ldots, n-1\}$, if each $(n-p-1)$-extreme support plane of $K$ supports $E$ [17, pp. 75-76]. Here a support plane is said to be $p$-extreme if its outer normal vector is a $p$-extreme normal vector of $K$. For 
further characterizations and properties of $p$-tangential bodies we refer to [17, Section 2.2].

So a 0 -tangential body of $E$ is the body $E$ itself and each $p$-tangential body of $E$ is also a $q$-tangential body for $p<q \leq n-1$. Notice also that there exist $p$-tangential bodies of $E$ which are not $(p-1)$-tangential bodies of $E$ (see e.g. [5, p. 163] or [9, Proof Th. 1.2]). A 1-tangential body is usually called cap-body, and it can be seen as the convex hull of $E$ and countably many points such that the line segment joining any pair of those points intersects $E$ (see [17, p. 76]). An $(n-1)$-tangential body will be briefly called a tangential body.

Notice that the form body $K^{*}$ of a convex body $K$ is always a tangential body. The following theorem shows the close relation existing between the inner parallel bodies and the tangential bodies.

Theorem 2.1 (Schneider [17, pp. 136-137]). Let $K \in \mathcal{K}_{0}^{n}$ and $-\mathrm{r}<\lambda<0$. Then $K \sim|\lambda| E$ is homothetic to $K$ if, and only if, $K$ is homothetic to a tangential body of $E$.

From now on we will write $K_{\lambda}$ to denote the (relative) inner/outer parallel bodies of $K$, i.e.,

$$
K_{\lambda}:= \begin{cases}K \sim|\lambda| E & \text { for }-\mathrm{r} \leq \lambda \leq 0 \\ K+\lambda E & \text { for } 0 \leq \lambda<\infty\end{cases}
$$

In [14, Lemma 4.8] it is proved that for $K \in \mathcal{K}_{0}^{n}$ and $-\mathrm{r} \leq \lambda \leq 0$ it holds

$$
K_{\lambda}+|\lambda| K^{*} \subseteq K \text {. }
$$

Here we characterize the convex bodies for which equality holds in (2.2).

Theorem 2.2. Let $K \in \mathcal{K}_{0}^{n}$ and let $E \in \mathcal{K}_{0}^{n}$ be regular. Then $K=K_{\lambda}+$ $|\lambda| K^{*}$ for every $-\mathrm{r} \leq \lambda \leq 0$ if and only if $K$ is a tangential body of $K_{-\mathrm{r}}+\mathrm{r} E$ satisfying that for all $-\mathrm{r} \leq \lambda \leq 0$,

$$
\mathcal{U}_{0}(K)=\mathcal{U}_{0}\left(K_{\lambda}+K^{*}\right) .
$$

The above theorem allows us to determine the extremal sets in the announced inequalities involving the quermassintegrals of inner parallel bodies:

Theorem 2.3. Let $K \in \mathcal{K}_{0}^{n}$ and let $E \in \mathcal{K}_{0}^{n}$ be regular and strictly convex. For $-\mathrm{r} \leq \lambda \leq 0$ and $i=0, \ldots, n-1$,

$$
\mathrm{W}_{i}\left(K_{\lambda} ; E\right) \leq \mathrm{W}_{i}(K ; E)-|\lambda| \sum_{k=0}^{n-i-1} \mathrm{~V}\left(K_{\lambda}[k], K[n-i-k-1], K^{*}, E[i]\right) .
$$

If $K$ is a tangential body of $K_{-\mathrm{r}}+\mathrm{r} E$ satisfying condition (2.3) then equality holds in all the inequalities. Conversely, if equality holds in (2.4) for some $i \in\{0, \ldots, n-1\}$ then $K$ is a tangential body of $K_{-\mathrm{r}}+\mathrm{r} E$.

These results improve previous bounds obtained in [3], where conditions for the equality case are not provided: 
Theorem 2.4 ([3, Theorem 2]). Let $K \in \mathcal{K}^{n}$. For $-\mathrm{r} \leq \lambda \leq 0$ and for any $i=0, \ldots, n-1$ it holds

$$
\mathrm{W}_{i}\left(K_{\lambda} ; E\right) \leq \mathrm{W}_{i}(K ; E)-|\lambda| \sum_{k=0}^{n-i-1} \mathrm{~V}\left(K_{\lambda}[k], K[n-i-k-1], E[i+1]\right) .
$$

Under the assumption that the convex body $K \in \mathcal{K}_{0}^{n}$ satisfies some particular "differentiability conditions", we can improve the previous inequalities. We will deal with it in the last section, where the so called classes $\mathcal{R}_{p}$, in which the convex bodies are classified, will be defined.

\section{0-EXTREME VeCtors of INNER PARALlel Bodies. The ProOF OF ThEOREM 2.2}

We start this section by studying some particular behavior of the inner parallel bodies with respect to their 0-extreme normal vectors, which will be needed further on.

In [14, Lemma 4.5] it is shown that for any $K \in \mathcal{K}^{n}$ and $-\mathrm{r}<\lambda \leq 0$ then

$$
\mathcal{U}_{0}\left(K_{\lambda}\right) \subseteq \mathcal{U}_{0}(K)
$$

A first question arising from the above relation is for which convex bodies the equality holds in (3.1). Before answering this question we prove a useful property regarding 0-extreme vectors and the Minkowski sum. In [14, Lemma 2.4] it is proved that for any $K, L \in \mathcal{K}^{n}$,

$$
\mathcal{U}_{0}(K) \cup \mathcal{U}_{0}(L) \subseteq \mathcal{U}_{0}(K+L)
$$

Next we show that the set of 0 -extreme vectors of the Minkowski sum of two convex bodies does not depend on the size of the summands involved in it.

Lemma 3.1. Let $K, L \in \mathcal{K}^{n}$ and $\lambda>0$. Then

$$
\mathcal{U}_{0}(K+L)=\mathcal{U}_{0}(K+\lambda L) .
$$

Proof. First we assume that $0<\lambda \leq 1$. Then, $K+L=K+\lambda L+(1-\lambda) L$, i.e., $K+\lambda L$ is the inner parallel body of $K+L$ with respect to $L$ at distance $1-\lambda$. So, by (3.1) we get $\mathcal{U}_{0}(K+\lambda L) \subseteq \mathcal{U}_{0}(K+L)$. On the other hand, it is clear that $\mathcal{U}_{0}(\lambda K)=\mathcal{U}_{0}(K)$ and hence $\mathcal{U}_{0}(\lambda K+\lambda L)=\mathcal{U}_{0}(K+L)$. Since $K+\lambda L=\lambda K+\lambda L+(1-\lambda) K$, we get that $\lambda K+\lambda L$ is the inner parallel body of $K+L$ with respect to $K$ at distance $1-\lambda$ and thus, using again (3.1), it follows that

$$
\mathcal{U}_{0}(K+L)=\mathcal{U}_{0}(\lambda K+\lambda L) \subseteq \mathcal{U}_{0}(K+\lambda L) .
$$

Together with the previous inclusion we get the result, i.e., $\mathcal{U}_{0}(K+L)=$ $\mathcal{U}_{0}(K+\lambda L)$.

Finally, if $\lambda \geq 1$ (and hence $1 / \lambda \leq 1)$ it is enough to consider $(1 / \lambda)(K+$ $\lambda L)=(1 / \lambda) K+L$. Since

$$
\mathcal{U}_{0}(K+\lambda L)=\mathcal{U}_{0}((1 / \lambda)(K+\lambda L))=\mathcal{U}_{0}((1 / \lambda) K+L)
$$


we have just to apply the previous case, interchanging the roles of $K$ and $L$, to obtain the result.

Now we deal with the equality case in (3.1).

Lemma 3.2. Let $K \in \mathcal{K}^{n}$ and let $E \in \mathcal{K}_{0}^{n}$ be regular. If $K$ is a tangential body of the outer parallel body $\left(K_{-\mathrm{r}}\right)_{\mathrm{r}}=K_{-\mathrm{r}}+\mathrm{r} E$, then for any $-\mathrm{r}<\lambda \leq 0$,

$$
\mathcal{U}_{0}(K)=\mathcal{U}_{0}\left(K_{\lambda}\right) \text {. }
$$

Proof. First we show that for every $u \in \mathcal{U}_{0}(K), h\left(K_{\lambda}, u\right)=h\left(\left(K_{-\mathrm{r}}\right)_{\mathrm{r}+\lambda}, u\right)$. Notice, on one hand, that since $K$ is a tangential body of $\left(K_{-\mathrm{r}}\right)_{\mathrm{r}}$ then $h(K, u)=h\left(\left(K_{-\mathrm{r}}\right)_{\mathrm{r}}, u\right)$ for any $u \in \mathcal{U}_{0}(K)$ and thus

$$
\begin{aligned}
h\left(K_{\lambda}, u\right) & \leq h(K, u)-|\lambda| h(E, u)=h\left(K_{-\mathrm{r}}+\mathrm{r} E, u\right)-|\lambda| h(E, u) \\
& =h\left(K_{-\mathrm{r}}, u\right)+(\mathrm{r}+\lambda) h(E, u) .
\end{aligned}
$$

On the other hand, it is clear from the definition of inner parallel body that since

$$
\left(K_{-\mathrm{r}}\right)_{\mathrm{r}+\lambda}+|\lambda| E=K_{-\mathrm{r}}+(\mathrm{r}-|\lambda|) E+|\lambda| E=K_{-\mathrm{r}}+\mathrm{r} E=\left(K_{-\mathrm{r}}\right)_{\mathrm{r}} \subseteq K,
$$

then $\left(K_{-\mathrm{r}}\right)_{\mathrm{r}+\lambda} \subseteq K_{\lambda}$ and hence we have

$$
h\left(K_{\lambda}, u\right) \geq h\left(K_{-\mathrm{r}}, u\right)+(\mathrm{r}+\lambda) h(E, u) .
$$

Thus we obtain the equality $h\left(K_{\lambda}, u\right)=h\left(\left(K_{-\mathrm{r}}\right)_{\mathrm{r}+\lambda}, u\right)$ for any $u \in \mathcal{U}_{0}(K)$, as required.

Now, in order to prove that $\mathcal{U}_{0}\left(K_{\lambda}\right)=\mathcal{U}_{0}(K)$ for every $-\mathrm{r}<\lambda \leq 0$, let $u \in \mathcal{U}_{0}(K)$. Since it holds that $h\left(K_{\lambda}, u\right)=h\left(\left(K_{-\mathrm{r}}\right)_{\mathrm{r}+\lambda}, u\right)$, the supporting hyperplanes $H\left(K_{\lambda}, u\right)$ and $H\left(\left(K_{-\mathrm{r}}\right)_{\mathrm{r}+\lambda}, u\right)$ coincide. Moreover, we know that $\left(K_{-\mathrm{r}}\right)_{\mathrm{r}+\lambda} \subseteq K_{\lambda}$. So, at any common point $x$ in the (non-empty) intersection of the support sets $K_{\lambda} \cap H\left(K_{\lambda}, u\right)$ and $\left(K_{-\mathrm{r}}\right)_{\mathrm{r}+\lambda} \cap H\left(\left(K_{-\mathrm{r}}\right)_{\mathrm{r}+\lambda}, u\right)$, the corresponding normal cones verify that $N\left(K_{\lambda}, x\right) \subseteq N\left(\left(K_{-\mathrm{r}}\right)_{\mathrm{r}+\lambda}, x\right)$. On the other hand, since clearly $\left(K_{-\mathrm{r}}\right)_{\mathrm{r}+\lambda}$ is regular then $\operatorname{dim} N\left(\left(K_{-\mathrm{r}}\right)_{\mathrm{r}+\lambda}, x\right)=1$; hence $\operatorname{dim} N\left(K_{\lambda}, x\right)=1$ which proves that $u \in \mathcal{U}_{0}\left(K_{\lambda}\right)$. Thus $\mathcal{U}_{0}(K) \subseteq$ $\mathcal{U}_{0}\left(K_{\lambda}\right)$ and with (3.1) we get the result.

Lemma 3.3. Let $K \in \mathcal{K}^{n}$ be a regular convex body. Then $\mathcal{U}_{0}(K)=\mathcal{U}_{0}\left(K_{\lambda}\right)$ for any $-\mathrm{r}<\lambda \leq 0$ if and only if $K=K_{-\mathrm{r}}+\mathrm{r} E$.

Proof. If $K=K_{-\mathrm{r}}+\mathrm{r} E$ then $K_{\lambda}=K_{-\mathrm{r}}+(\mathrm{r}-|\lambda|) E$, for any $-\mathrm{r}<\lambda \leq 0$, and applying Lemma 3.1 we obtain

$$
\mathcal{U}_{0}\left(K_{\lambda}\right)=\mathcal{U}_{0}\left(K_{-\mathrm{r}}+(\mathrm{r}+\lambda) E\right)=\mathcal{U}_{0}\left(K_{-\mathrm{r}}+\mathrm{r} E\right)=\mathcal{U}_{0}(K)
$$

(notice that in this part of the result, the regularity of the convex body $K$ is not needed). So, we assume $\mathcal{U}_{0}\left(K_{\lambda}\right)=\mathcal{U}_{0}(K)$ for all $\lambda \in(-\mathrm{r}, 0]$. It is known that (see [14, Lemma 4.4])

$$
\text { if } u \in \mathcal{U}_{0}\left(K_{\lambda}\right) \text { then } h\left(K_{\lambda}, u\right)=h(K, u)+\lambda h(E, u) .
$$


Since $K$ is regular, $\mathcal{U}_{0}\left(K_{\lambda}\right)=\mathcal{U}_{0}(K)=\mathbb{S}^{n-1}$ and hence, for all $u \in \mathbb{S}^{n-1}=$ $\mathcal{U}_{0}\left(K_{\lambda}\right)$ it holds $h\left(K_{\lambda}, u\right)=h(K, u)+\lambda h(E, u)$. Therefore (see (2.1))

$$
\begin{aligned}
\mathrm{W}_{n-1}( & \left.K_{\lambda} ; E\right)=\frac{1}{n} \int_{\mathbb{S}^{n-1}} h\left(K_{\lambda}, u\right) d \mathrm{~S}_{n-1}(E ; u) \\
& =\frac{1}{n} \int_{\mathbb{S}^{n-1}} h(K, u) d \mathrm{~S}_{n-1}(E ; u)+\frac{1}{n} \int_{\mathbb{S}^{n-1}} \lambda h(E, u) d \mathrm{~S}_{n-1}(E ; u) \\
& =\mathrm{W}_{n-1}(K ; E)+\lambda \mathrm{W}_{n-1}(E ; E)=\mathrm{W}_{n-1}(K ; E)-|\lambda| \mathrm{W}_{n-1}(E ; E) .
\end{aligned}
$$

Thus by the linearity of mixed volumes (see e.g. [17, p. 279]) we get

$$
\mathrm{W}_{n-1}(K ; E)=\mathrm{W}_{n-1}\left(K_{\lambda} ; E\right)+|\lambda| \mathrm{W}_{n-1}(E ; E)=\mathrm{W}_{n-1}\left(K_{\lambda}+|\lambda| E\right),
$$

and since it always holds $K_{\lambda}+|\lambda| E \subseteq K$, we conclude that $K=K_{\lambda}+|\lambda| E$. Notice that we have shown $K=K_{\lambda}+|\lambda| E$ for all $\lambda \in(-\mathrm{r}, 0]$, which implies that $K=K_{-\mathrm{r}}+\mathrm{r} E$, as required.

Now we prove Theorem 2.2. The following result can be found in 14, Lemma 4.9], and it will be needed in the proof of the theorem. We will write $K_{\lambda}^{*}=\left(K_{\lambda}\right)^{*}$ to denote the form body of the inner parallel body of $K$ at distance $|\lambda|$.

Lemma 3.4. Let $K \in \mathcal{K}^{n}$. For any $u \in \mathbb{S}^{n-1}$, the derivative of $h(\lambda, u)=$ $h\left(K_{\lambda}, u\right)$ with respect to $\lambda \in(-\mathrm{r}, \infty)$ exists almost everywhere and

$$
\frac{d}{d \lambda} h(\lambda, u) \geq h\left(K_{\lambda}^{*}, u\right)
$$

Equality holds for every $-\mathrm{r}<\lambda<\infty$ if

$$
\operatorname{cl} \mathcal{U}_{0}\left(K_{\lambda}\right)=\mathcal{U}_{0}\left(K_{\lambda}+K_{\lambda}^{*}\right)
$$

for every $-\mathrm{r}<\lambda<\infty$.

Proof of Theorem 2.2. We start by assuming that $K=K_{\lambda}+|\lambda| K^{*}$ for every $-\mathrm{r} \leq \lambda \leq 0$, in particular, that $K=K_{-\mathrm{r}}+\mathrm{r} K^{*}$, and we prove that $K$ is a tangential body of $K_{-\mathrm{r}}+\mathrm{r} E$. In order to do that, we first show that $K_{-\mathrm{r}}+(\mathrm{r}+\lambda) K^{*}$ is the inner parallel body of $K$ at distance $|\lambda|$, i.e., $K_{\lambda}=K_{-\mathrm{r}}+(\mathrm{r}+\lambda) K^{*}$, for any $-\mathrm{r} \leq \lambda \leq 0$.

Since $\mathcal{U}_{0}\left(K_{\lambda}\right) \subseteq \mathcal{U}_{0}(K)$ (see (3.1)) then it always holds $K_{\lambda}^{*} \supseteq K^{*}$ for any $-\mathrm{r}<\lambda \leq 0$. Thus we get from (2.2) that, for $-\mathrm{r}<\lambda \leq 0$,

$$
K_{\lambda} \supseteq\left(K_{\lambda}\right)_{-\mathrm{r}\left(K_{\lambda} ; E\right)}+\mathrm{r}\left(K_{\lambda} ; E\right) K_{\lambda}^{*}=K_{-\mathrm{r}}+(\mathrm{r}+\lambda) K_{\lambda}^{*} \supseteq K_{-\mathrm{r}}+(\mathrm{r}+\lambda) K^{*} .
$$

Since for $\lambda=-\mathrm{r}$ we get a trivial identity, the inclusion $K_{\lambda} \supseteq K_{-\mathrm{r}}+(\mathrm{r}+\lambda) K^{*}$ is proved for the full interval $[-\mathrm{r}, 0]$. So it remains to show the reverse inclusion in the particular case when $K=K_{-\mathrm{r}}+\mathrm{r} K^{*}$. Notice that

$$
K_{\lambda}=\left(K_{-\mathrm{r}}+\mathrm{r} K^{*}\right)_{\lambda}=\bigcap_{u \in \mathcal{U}_{0}(K)}\left\{x:\langle x, u\rangle \leq h\left(K_{-\mathrm{r}}+\mathrm{r} K^{*}, u\right)-|\lambda| h(E, u)\right\} .
$$


On the other hand, $\mathcal{U}_{0}(K)=\mathcal{U}_{0}\left(K_{-\mathrm{r}}+\mathrm{r} K^{*}\right)=\mathcal{U}_{0}\left(K_{-\mathrm{r}}+(\mathrm{r}+\lambda) K^{*}\right)$ for $-\mathrm{r}<\lambda \leq 0$ by Lemma 3.1. Hence we can write

$$
\begin{aligned}
K_{-\mathrm{r}}+(\mathrm{r}+\lambda) K^{*} & =\bigcap_{u \in \mathcal{U}_{0}\left(K_{-\mathrm{r}}+(\mathrm{r}+\lambda) K^{*}\right)}\left\{x:\langle x, u\rangle \leq h\left(K_{-\mathrm{r}}+(\mathrm{r}+\lambda) K^{*}, u\right)\right\} \\
& =\bigcap_{u \in \mathcal{U}_{0}(K)}\left\{x:\langle x, u\rangle \leq h\left(K_{-\mathrm{r}}+(\mathrm{r}+\lambda) K^{*}, u\right)\right\}
\end{aligned}
$$

Thus if $x \in K_{\lambda},-\mathrm{r}<\lambda \leq 0$, it lies in the intersection given in (3.6), and in order to show that $x \in K_{-\mathrm{r}}+(\mathrm{r}+\lambda) K^{*}$ we have to prove that it lies also in (3.7). So for any $u \in \mathcal{U}_{0}(K)$ it follows

$$
\begin{aligned}
\langle x, u\rangle & \leq h\left(K_{-\mathrm{r}}+\mathrm{r} K^{*}, u\right)-|\lambda| h(E, u)=h\left(K_{-\mathrm{r}}, u\right)+\mathrm{r} h\left(K^{*}, u\right)-|\lambda| h\left(K^{*}, u\right) \\
& =h\left(K_{-\mathrm{r}}, u\right)+(\mathrm{r}+\lambda) h\left(K^{*}, u\right)=h\left(K_{-\mathrm{r}}+(\mathrm{r}+\lambda) K^{*}, u\right),
\end{aligned}
$$

i.e., $x \in K_{-\mathrm{r}}+(\mathrm{r}+\lambda) K^{*}$, which shows that $K_{\lambda} \subseteq K_{-\mathrm{r}}+(\mathrm{r}+\lambda) K^{*}$ for $-\mathrm{r}<\lambda \leq 0$. The case $\lambda=-\mathrm{r}$ holds trivially.

Thus we have shown that

$$
K_{\lambda}=\left(K_{-\mathrm{r}}+\mathrm{r} K^{*}\right)_{\lambda}=K_{-\mathrm{r}}+(\mathrm{r}+\lambda) K^{*}
$$

in the full range $-\mathrm{r} \leq \lambda \leq 0$ and on account of Lemma 3.1, it follows that

$$
\mathcal{U}_{0}\left(K_{\lambda}\right)=\mathcal{U}_{0}\left(K_{-\mathrm{r}}+(\mathrm{r}+\lambda) K^{*}\right)=\mathcal{U}_{0}\left(K_{-\mathrm{r}}+\mathrm{r} K^{*}\right)=\mathcal{U}_{0}(K) .
$$

We assume now that $K$ is not a tangential body of $\left(K_{-\mathrm{r}}\right)_{\mathrm{r}}=K_{-\mathrm{r}}+\mathrm{r} E$. Then by definition of tangential body there exists $u_{0} \in \mathcal{U}_{0}(K)$ such that

$$
H\left(\left(K_{-\mathrm{r}}\right)_{\mathrm{r}}, u_{0}\right) \cap H\left(K, u_{0}\right)=\emptyset ;
$$

in particular, we have that the distance, say $\mu$, between the above two hyperplanes is strictly positive. On the other hand, since $u_{0} \in \mathcal{U}_{0}(K)=$ $U_{0}\left(K_{\lambda}\right)$ we know $($ see $(\overline{3.4}))$ that $h\left(K_{\lambda}, u_{0}\right)=h\left(K, u_{0}\right)-|\lambda| h\left(E, u_{0}\right)$ for every $-\mathrm{r} \leq \lambda \leq 0$, and hence the distance between the parallel hyperplanes $H\left(K, u_{0}\right)$ and $H\left(K_{\lambda}, u_{0}\right)$ is $|\lambda| h\left(E, u_{0}\right)$. Moreover, the distance between the (parallel) hyperplanes $H\left(\left(K_{-\mathrm{r}}\right)_{\mathrm{r}+\lambda}, u_{0}\right)$ and $H\left(\left(K_{-\mathrm{r}}\right)_{\mathrm{r}}, u_{0}\right)$ is also $|\lambda| h\left(E, u_{0}\right)$ since the body $\left(K_{-\mathrm{r}}\right)_{\mathrm{r}}=K_{-\mathrm{r}}+\mathrm{r} E$ is just the outer parallel body of $\left(K_{-\mathrm{r}}\right)_{\mathrm{r}+\lambda}=K_{-\mathrm{r}}+(\mathrm{r}+\lambda) E=K_{-\mathrm{r}}+(\mathrm{r}-|\lambda|) E$ at distance $|\lambda|$, for every $\lambda$. Thus, the distance between $H\left(K_{\lambda}, u_{0}\right)$ and $H\left(\left(K_{-\mathrm{r}}\right)_{\mathrm{r}+\lambda}, u_{0}\right)$ is $\mu>0$, for every $\lambda$. But this leads to a contradiction, since when $|\lambda| \rightarrow \mathrm{r}$, the distance between $H\left(K_{\lambda}, u_{0}\right)$ and $H\left(\left(K_{-\mathrm{r}}\right)_{\mathrm{r}+\lambda}, u_{0}\right)$ goes to zero.

Thus we already know that $K$ is a tangential body of $\left(K_{-r}\right)_{r}$, and it remains to prove that $K$ satisfies condition (2.3). But it is a direct consequence of Lemma 3.1; for all $-\mathrm{r} \leq \lambda \leq 0$,

$$
\mathcal{U}_{0}(K)=\mathcal{U}_{0}\left(K_{\lambda}+|\lambda| K^{*}\right)=\mathcal{U}_{0}\left(K_{\lambda}+K^{*}\right) .
$$

Conversely, now we assume that $K$ is a tangential body of $\left(K_{-\mathrm{r}}\right)_{\mathrm{r}}=$ $K_{-\mathrm{r}}+\mathrm{r} E$ satisfying (2.3) for all $-\mathrm{r} \leq \lambda \leq 0$. Since $K$ is a tangential body of $\left(K_{-\mathrm{r}}\right)_{\mathrm{r}}$, Lemma 3.2 ensures that $\mathcal{U}_{0}\left(K_{\lambda}\right)=\mathcal{U}_{0}(K)$ for every $-\mathrm{r}<\lambda \leq 0$ and 
hence $K^{*}=K_{\lambda}^{*}$ for $-\mathrm{r}<\lambda \leq 0$. We work first in the half-opened interval $(-\mathrm{r}, 0]$. It is known (see [10, Lemma 2.1]) that

$$
\mathcal{U}_{0}\left(K^{*}\right)=\operatorname{cl} \mathcal{U}_{0}(K) \quad \text { when } E \text { is regular, }
$$

and with (3.2) we get

$$
\mathcal{U}_{0}(K)=\mathcal{U}_{0}\left(K_{\lambda}+K^{*}\right) \supseteq \mathcal{U}_{0}\left(K_{\lambda}\right) \cup \mathcal{U}_{0}\left(K^{*}\right)=\mathcal{U}_{0}(K) \cup \operatorname{cl} \mathcal{U}_{0}(K) \supseteq \mathcal{U}_{0}(K) .
$$

Therefore, in particular, $\mathcal{U}_{0}(K)$ is closed and $\operatorname{cl} \mathcal{U}_{0}(K)=\mathcal{U}_{0}\left(K_{\lambda}+K^{*}\right)$. Thus, the above properties allow us to conclude that

$$
\operatorname{cl} \mathcal{U}_{0}\left(K_{\lambda}\right)=\operatorname{cl} \mathcal{U}_{0}(K)=\mathcal{U}_{0}\left(K_{\lambda}+K^{*}\right)=\mathcal{U}_{0}\left(K_{\lambda}+K_{\lambda}^{*}\right)
$$

i.e., we get condition (3.5). Then Lemma 3.4 ensures that for all $\lambda \in(-\mathrm{r}, 0]$ and $u \in \mathbb{S}^{n-1}$,

$$
\frac{d}{d \lambda} h(\lambda, u)=h\left(K_{\lambda}^{*}, u\right)=h\left(K^{*}, u\right) .
$$

Now we fix $u \in \mathbb{S}^{n-1}$ and define the function

$$
f(\lambda)=h(K, u)-h(\lambda, u)+\lambda h\left(K^{*}, u\right),
$$

which is absolutely continuous (since $h(\lambda, u)$ is concave in $\lambda$, see [6, Theorem 1.1]), almost everywhere differentiable by Lemma 3.4 and clearly verifies that $f^{\prime}(\lambda)=0$. Thus $f$ is a constant function and since $f(0)=0$ we obtain $f \equiv 0$, i.e.,

$h(K, u)=h(\lambda, u)-\lambda h\left(K^{*}, u\right)=h\left(K_{\lambda}, u\right)+|\lambda| h\left(K^{*}, u\right)=h\left(K_{\lambda}+|\lambda| K^{*}, u\right)$, for all $u \in \mathbb{S}^{n-1}$. Hence $K=K_{\lambda}+|\lambda| K^{*}$ for every $\lambda \in(-\mathrm{r}, 0]$. Now, for each $u \in \mathbb{S}^{n-1}$ fixed, since $h(K, u)=h\left(K_{\lambda}+|\lambda| K^{*}, u\right)=h\left(K_{\lambda}, u\right)+|\lambda| h\left(K^{*}, u\right)$ for every $\lambda \in(-\mathrm{r}, 0]$, taking limits when $\lambda$ tends to $-\mathrm{r}$ and taking into account that the support function is continuous with respect to the Hausdorff metric, we also get that $h(K, u)=h\left(K_{-\mathrm{r}}, u\right)+\mathrm{r} h\left(K^{*}, u\right)$ for all $u \in \mathbb{S}^{n-1}$. Hence $K=K_{\lambda}+|\lambda| K^{*}$ for every $\lambda \in[-\mathrm{r}, 0]$ and it concludes the proof.

Remark 3.1. It is enough to assume $K=K_{-\mathrm{r}}+\mathrm{r} K^{*}$ in the statement of Theorem 2.2, since it is equivalent to the condition $K=K_{\lambda}+|\lambda| K^{*}$ for all $\lambda \in[-\mathrm{r}, 0]$ : clearly one direction is trivial; for the converse just notice that if $K=K_{-\mathrm{r}}+\mathrm{r} K^{*}$ then $K_{\lambda}=K_{-\mathrm{r}}+(\mathrm{r}+\lambda) K^{*}$ (see (3.8)), and hence, for all $\lambda \in[-\mathrm{r}, 0]$,

$$
K_{\lambda}+|\lambda| K^{*}=K_{-\mathrm{r}}+(\mathrm{r}-|\lambda|) K^{*}+|\lambda| K^{*}=K_{-\mathrm{r}}+\mathrm{r} K^{*}=K .
$$

We have settled the theorem in this way in order to establish the precise characterization of the equality case in (2.2).

Remark 3.2. Condition (2.3) cannot be omitted: let $\sigma$ be a line segment of length $\ell \geq 2$ in $\mathbb{R}^{3}$ and take a point $x$ lying outside the solid cylinder with circular cross section of radius 1 and axis the line aff $\sigma$. The convex body $K$ obtained as the convex hull $K=\operatorname{conv}\left\{\sigma+B^{3}, x\right\}$ (see Figure 2) satisfies:

- $\operatorname{ker} K=\sigma$ and $\mathrm{r}\left(K ; B^{3}\right)=1$;

- $K$ is a 1-tangential body of $\sigma+B^{3}=K_{-1}+B^{3}$; 
- $K^{*}$ is just the convex hull of $B^{3}$ and a suitable segment;

- condition (2.3) does not hold for any $\lambda$;

hence $K \neq K_{\lambda}+|\lambda| K^{*}$.

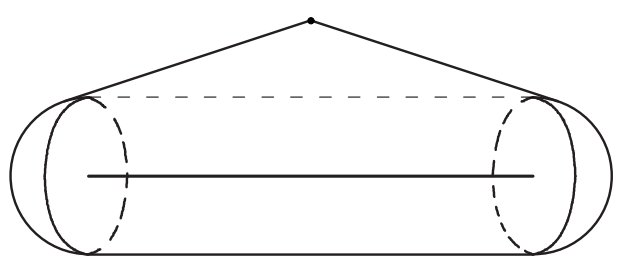

Figure 2. A tangential body of $K_{-\mathrm{r}}+\mathrm{r} B^{3}$ not satisfying (2.3).

Remark 3.3. Notice that for $E$ regular, if $K=K_{\lambda}+|\lambda| K^{*}$ for every $-\mathrm{r} \leq \lambda \leq 0$ then

$$
\frac{d}{d \lambda} h(\lambda, u)=h\left(K_{\lambda}^{*}, u\right)=h\left(K^{*}, u\right)
$$

for all $u \in \mathbb{S}^{n-1}$.

\section{Bounding THE QUERMASSINTEgRALS OF THE INNER PARALLEL BOdY}

In this section we get some bounds for the relative quermassintegrals of the inner parallel body in terms of the ones of the original body $K$. The proof of the inequality in Theorem 2.3 follows the same idea as the one in [3, Theorem 2]; the equality case is however completely new.

Proof of Theorem 2.3. Using (2.2) and the monotonicity and linearity of mixed volumes (see e.g. [17, p. 277, p.279]) we get that for $-\mathrm{r} \leq \lambda \leq 0$,

$$
\begin{aligned}
\mathrm{W}_{i}(K ; E)=\mathrm{V}(K[n-i], E[i]) \geq \mathrm{V}\left(K_{\lambda}+|\lambda| K^{*}, K[n-i-1], E[i]\right) \\
=\mathrm{V}\left(K_{\lambda}, K[n-i-1], E[i]\right)+|\lambda| \mathrm{V}\left(K^{*}, K[n-i-1], E[i]\right) \\
\geq \mathrm{V}\left(K_{\lambda}, K_{\lambda}+|\lambda| K^{*}, K[n-i-2], E[i]\right)+|\lambda| \mathrm{V}\left(K^{*}, K[n-i-1], E[i]\right) \\
\geq \mathrm{V}\left(K_{\lambda}, K_{\lambda}, K[n-i-2], E[i]\right)+|\lambda| \mathrm{V}\left(K_{\lambda}, K^{*}, K[n-i-2], E[i]\right) \\
\quad+|\lambda| \mathrm{V}\left(K^{*}, K[n-i-1], E[i]\right) \geq \ldots \\
\geq \mathrm{W}_{i}\left(K_{\lambda} ; E\right)+|\lambda| \sum_{k=0}^{n-i-1} \mathrm{~V}\left(K_{\lambda}[k], K^{*}, K[n-i-k-1], E[i]\right) .
\end{aligned}
$$

Now we deal with the equality case. If $K$ is a tangential body of $K_{-\mathrm{r}}+\mathrm{r} E$ satisfying condition (2.3), Theorem 2.2 ensures that $K=K_{\lambda}+|\lambda| K^{*}$ for every $-\mathrm{r} \leq \lambda \leq 0$, and hence equality holds in (2.4). Conversely, now we assume that equality holds in (2.4) for some $i \in\{0, \ldots, n-1\}$. Then we have, in particular, that

$$
\mathrm{V}(K[n-i], E[i])=\mathrm{V}\left(K_{\lambda}+|\lambda| K^{*}, K[n-i-1], E[i]\right),
$$


or equivalently, using the formula for the mixed volumes given in (2.1) we get that

$$
\begin{aligned}
\int_{\mathbb{S}^{n-1}} h(K, u) d \mathrm{~S}(K[n-i-1], E[i] ; u) \\
=\int_{\mathbb{S}^{n-1}} h\left(K_{\lambda}+|\lambda| K^{*}, u\right) d \mathrm{~S}(K[n-i-1], E[i] ; u) .
\end{aligned}
$$

Since $K_{\lambda}+|\lambda| K^{*} \subseteq K$ (see (2.2)), we get that the above identity for the integrals is equivalent to

$$
h\left(K_{\lambda}+|\lambda| K^{*}, u\right)=h(K, u) \quad \text { for all } u \in \operatorname{supp} \mathrm{S}(K[n-i-1], E[i] ; u) .
$$

On the other hand, since $E$ is regular and strictly convex, results by Schneider (see [16, pp. 135-136]) show that $\operatorname{supp} \mathrm{S}(K[n-i-1], E[i] ; u)=\operatorname{cl}_{i}(K)$ $\supseteq \operatorname{cl} \mathcal{U}_{0}(K)$. So we get $h\left(K_{\lambda}+|\lambda| K^{*}, u\right)=h(K, u)$ for all $u \in \operatorname{cl} \mathcal{U}_{0}(K)$. Notice that it implies, in particular, that $K$ is a tangential body of $K_{\lambda}+|\lambda| K^{*}$.

Now observe that, for every $u \in \operatorname{cl} \mathcal{U}_{0}(K)=\mathcal{U}_{0}\left(K^{*}\right)$ (cf. (3.9)) it holds

$$
\begin{aligned}
h(K, u) & =h\left(K_{\lambda}+|\lambda| K^{*}, u\right)=h\left(K_{\lambda}, u\right)+|\lambda| h\left(K^{*}, u\right) \\
& =h\left(K_{\lambda}, u\right)+|\lambda| h(E, u)=h\left(K_{\lambda}+|\lambda| E, u\right),
\end{aligned}
$$

which shows that $K$ is a tangential body of $K_{\lambda}+|\lambda| E$, for all $\lambda \in[-\mathrm{r}, 0]$; in particular, $K$ is a tangential body of $K_{-\mathrm{r}}+\mathrm{r} E$, as required.

Notice that the assumptions of regularity and strict convexity for $E$ are needed just for the equality case; the inequalities hold for any $E \in \mathcal{K}_{0}^{n}$.

The particular case $i=0$ provides a new upper bound for the volume of the inner parallel body.

Corollary 4.1. Let $K \in \mathcal{K}_{0}^{n}$ and let $E \in \mathcal{K}_{0}^{n}$ be a regular and strictly convex body. For $-\mathrm{r} \leq \lambda \leq 0$ it holds

$$
\mathrm{V}\left(K_{\lambda}\right) \leq \mathrm{V}(K)-|\lambda| \sum_{k=0}^{n-1} \mathrm{~V}\left(K_{\lambda}[k], K[n-k-1], K^{*}\right) .
$$

If $K$ is a tangential body of $K_{-\mathrm{r}}+\mathrm{r} E$ satisfying condition (2.3) then equality holds. Conversely, if equality holds then $K$ is a tangential body of $K_{-\mathrm{r}}+\mathrm{r} E$.

Notice that in the case $\lambda=-r$ we get the following lower bound for the volume of $K$ :

$$
\mathrm{V}(K) \geq \mathrm{r} \sum_{k=0}^{n-1} \mathrm{~V}\left(K_{-\mathrm{r}}[k], K[n-k-1], K^{*}\right) .
$$

\section{Inequalities for CONVEX BODIES LYING IN THE Class $\mathcal{R}_{p}$}

The family of inner and outer parallel bodies of a convex body $K$ (with respect to $E$ ) determines a concave one-parameter family (see [17, p. 135]) of sets which allows us to consider quermassintegrals as functions depending 
on a real variable, $\mathrm{W}_{i}(\lambda)=\mathrm{W}_{i}\left(K_{\lambda}\right)$ for $-\mathrm{r} \leq \lambda \leq \infty$. The Brunn-Minkowski theorem for relative quermassintegrals (see e.g. [17, p. 339]) ensures that

$$
{ }^{\prime} \mathrm{W}_{i}(\lambda) \geq \mathrm{W}_{i}^{\prime}(\lambda) \geq(n-i) \mathrm{W}_{i+1}(\lambda)
$$

for $i=0, \ldots, n-1$, where ${ }^{\prime} \mathrm{W}_{i}$ and $\mathrm{W}_{i}^{\prime}$ denote, respectively, the left and right derivatives of the function $\mathrm{W}_{i}$. It is well known (see e.g. [1, 12]) that the volume is always differentiable and $\mathrm{V}^{\prime}(\lambda)=n \mathrm{~W}_{1}(\lambda)$. Moreover, if $\lambda \geq 0$ then it is clear from (1.2) that all quermassintegrals are differentiable at $\lambda$ (notice that in the case $\lambda=0$ we speak about differentiability from the right) and $\mathrm{W}_{i}^{\prime}(\lambda)=(n-i) \mathrm{W}_{i+1}(\lambda)$. The question arises for which convex bodies equalities hold in (5.1) for the full range $-\mathrm{r} \leq \lambda<\infty$.

Definition 5.1 ([10]). A convex body $K \in \mathcal{K}^{n}$ belongs to the class $\mathcal{R}_{p}$, $0 \leq p \leq n-1$, if for all $0 \leq i \leq p$, and for $-\mathrm{r} \leq \lambda<\infty$ it holds

$$
{ }^{\prime} \mathrm{W}_{i}(\lambda)=\mathrm{W}_{i}^{\prime}(\lambda)=(n-i) \mathrm{W}_{i+1}(\lambda) .
$$

Since $\mathrm{V}^{\prime}(\lambda)=n \mathrm{~W}_{1}(\lambda)$ then $\mathcal{R}_{0}=\mathcal{K}^{n}$. Moreover $\mathcal{R}_{i+1} \subset \mathcal{R}_{i}$ strictly for $i=0, \ldots, n-2$, see $[10$. The problem of determining the convex bodies belonging to the classes $\mathcal{R}_{p}$ was originally posed by Hadwiger [7] in $\mathbb{R}^{3}$ and for $E=B_{n}$. In [10] the general $n$-dimensional problem is studied.

Remark 5.1. Inequalities in Theorem 2.3 allow to give an alternative proof to the fact that the left derivative of the $i$-th quermassintegral with respect to $\lambda,-\mathrm{r}<\lambda \leq 0$ is bounded from below by $(n-i) \mathrm{V}\left(K_{\lambda}[n-i-1], K_{\lambda}^{*}, E[i]\right)$, which was proved in [14, Lemma 3.5]: for $h \geq 0$, using (2.4) and considering that $K_{\lambda-h}$ is an inner parallel body of $K_{\lambda}$ if $\lambda-h>-\mathrm{r}$, we get

$$
\begin{aligned}
{ }^{\prime} \mathrm{W}_{i}(\lambda) & =\lim _{h \rightarrow 0} \frac{\mathrm{W}_{i}\left(K_{\lambda} ; E\right)-\mathrm{W}_{i}\left(K_{\lambda-h} ; E\right)}{h} \\
& \geq \lim _{h \rightarrow 0} \frac{h \sum_{k=0}^{n-i-1} \mathrm{~V}\left(K_{\lambda-h}[k], K_{\lambda}[n-i-k-1], K_{\lambda}^{*}, E[i]\right)}{h} \\
& =\sum_{k=0}^{n-i-1} \mathrm{~V}\left(K_{\lambda}[n-i-1], K_{\lambda}^{*}, E[i]\right)=(n-i) \mathrm{V}\left(K_{\lambda}[n-i-1], K_{\lambda}^{*}, E[i]\right) .
\end{aligned}
$$

Moreover, since $E \subseteq K_{\lambda}^{*}$ for all $-\mathrm{r}<\lambda \leq 0$, we also get the bound given in (5.1), namely ${ }^{\prime} \mathrm{W}_{i}(\lambda) \geq(n-i) \mathrm{W}_{i+1}(\lambda)$.

Under the assumption that the convex body $K \in \mathcal{K}_{0}^{n}$ lies in the class $\mathcal{R}_{p}$, we can improve the inequalities in Theorem 2.3 .

Proposition 5.1. Let $K \in \mathcal{K}_{0}^{n}$ lie in the class $\mathcal{R}_{p}$ and let $E \in \mathcal{K}_{0}^{n}$ be regular and strictly convex. For $0 \leq i \leq p$ and for $-\mathrm{r} \leq \lambda \leq 0$ it holds

$$
\begin{aligned}
\mathrm{W}_{i}\left(K_{\lambda} ; E\right) & \geq \mathrm{W}_{i}(K ; E)-(n-i)|\lambda| \mathrm{W}_{i+1}(K ; E) \\
& +(n-i) \frac{\lambda^{2}}{2} \mathrm{~V}\left(K[n-i-2], K^{*}, E[i+1]\right) \\
& -(n-i) \sum_{k=1}^{n-i-2} \int_{\lambda}^{0} t \mathrm{~V}\left(K_{t}[k], K[n-i-k-2], K^{*}, E[i+1]\right) d t .
\end{aligned}
$$


If $K$ is a tangential body of $K_{-\mathrm{r}}+\mathrm{r} E$ satisfying condition (2.3) then equality holds in all the inequalities. Conversely, if equality holds in (5.3) for some $i \in\{0, \ldots, p\}$ then $K$ is a tangential body of $K_{-\mathrm{r}}+\mathrm{r} E$.

Proof. If we consider inequality (2.4) in the case of the $(i+1)$-th quermassintegral, $i=0, \ldots, p$,

$$
\begin{aligned}
\mathrm{W}_{i+1}\left(K_{\lambda} ; E\right) \leq & \mathrm{W}_{i+1}(K ; E)-|\lambda| \mathrm{V}\left(K[n-i-2], K^{*}, E[i+1]\right) \\
& -|\lambda| \sum_{k=1}^{n-i-2} \mathrm{~V}\left(K_{\lambda}[k], K[n-i-k-2], K^{*}, E[i+1]\right),
\end{aligned}
$$

integrating from $\lambda$ to 0 we get

$$
\begin{aligned}
\int_{\lambda}^{0} \mathrm{~W}_{i+1}\left(K_{t} ; E\right) d t & \leq \int_{\lambda}^{0}\left[\mathrm{~W}_{i+1}(K ; E)+t \mathrm{~V}\left(K[n-i-2], K^{*}, E[i+1]\right)\right] d t \\
& +\int_{\lambda}^{0} t \sum_{k=1}^{n-i-2} \mathrm{~V}\left(K_{t}[k], K[n-i-k-2], K^{*}, E[i+1]\right) d t
\end{aligned}
$$

Since $K \in \mathcal{R}_{p}$, we have $\mathrm{W}_{i}^{\prime}\left(K_{t}\right)=(n-i) \mathrm{W}_{i+1}\left(K_{t}\right)$ for $i=0, \ldots, p$, and hence

$$
\begin{array}{r}
\frac{\mathrm{W}_{i}(K ; E)-\mathrm{W}_{i}\left(K_{\lambda} ; E\right)}{n-i} \leq-\lambda \mathrm{W}_{i+1}(K ; E)-\frac{\lambda^{2}}{2} \mathrm{~V}\left(K[n-i-2], K^{*}, E[i+1]\right) \\
+\sum_{k=1}^{n-i-2} \int_{\lambda}^{0} t \mathrm{~V}\left(K_{t}[k], K[n-i-k-2], K^{*}, E[i+1]\right) d t
\end{array}
$$

which concludes the proof of the inequality. The conditions for the equality case follow directly from Theorem 2.3 .

Proposition 5.1 for the class $\mathcal{R}_{0}$ leads to the following corollary.

Corollary 5.1. Let $K \in \mathcal{K}_{0}^{n}$ and let $E \in \mathcal{K}_{0}^{n}$ be a regular and strictly convex body. For $-\mathrm{r} \leq \lambda \leq 0$ we have

$$
\begin{aligned}
\mathrm{V}\left(K_{\lambda}\right) \geq \mathrm{V}(K) & -n|\lambda| \mathrm{W}_{1}(K ; E)+n \frac{\lambda^{2}}{2} \mathrm{~V}\left(K[n-2], K^{*}, E\right) \\
& -n \sum_{k=1}^{n-2} \int_{\lambda}^{0} t \mathrm{~V}\left(K_{t}[k], K[n-k-2], K^{*}, E\right) d t .
\end{aligned}
$$

If $K$ is a tangential body of $K_{-\mathrm{r}}+\mathrm{r} E$ satisfying condition (2.3) then equality holds. Conversely, if equality holds then $K$ is a tangential body of $K_{-\mathrm{r}}+\mathrm{r} E$.

This inequality strengthens the one obtained by Brannen in 3, Corollary 2], namely,

$$
\begin{aligned}
\mathrm{V}\left(K_{\lambda}\right) \geq \mathrm{V}(K) & -n|\lambda| \mathrm{W}_{1}(K ; E)+n \frac{\lambda^{2}}{2} \mathrm{~W}_{2}(K ; E) \\
& +n \sum_{k=1}^{n-2} \int_{0}^{|\lambda|} t \mathrm{~V}\left(K_{t}[k], K[n-k-2], E[2]\right) d t .
\end{aligned}
$$


Notice that when $\lambda=-\mathrm{r}$, Corollary 5.1 provides an upper bound for the volume of $K$ :

$$
\begin{aligned}
\frac{1}{n} \mathrm{~V}(K) \leq \mathrm{rW}_{1}(K ; E) & -\frac{\mathrm{r}^{2}}{2} \mathrm{~V}\left(K[n-2], K^{*}, E\right) \\
& +\sum_{k=1}^{n-2} \int_{-\mathrm{r}}^{0} t \mathrm{~V}\left(K_{t}[k], K[n-k-2], K^{*}, E\right) d t .
\end{aligned}
$$

Moreover, in the case $n=3$, the above inequality is written as

$$
\frac{1}{3} \mathrm{~V}(K) \leq \mathrm{rW}_{1}(K ; E)-\frac{\mathrm{r}^{2}}{2} \mathrm{~V}\left(K, K^{*}, E\right)+\int_{-\mathrm{r}}^{0} t \mathrm{~V}\left(K_{t}, K^{*}, E\right) d t .
$$

It improves the corresponding inequality by Brannen (5.4) for $n=3$ which, in turn, is sharper than the so called Osserman inequality in the particular case of $n=3$ and $E=B^{3}$ (for a proof of this assertion see [3, p. 3982]), namely

$$
\mathrm{V}(K) \leq 3 \mathrm{r}(K) \mathrm{W}_{1}(K)-2 \mathrm{r}(K)^{2}\left[\mathrm{~V}\left(B^{3}\right) \mathrm{W}_{1}(K)\right]^{1 / 2}
$$

(see [13]); here we write, as usual in the literature, $\mathrm{W}_{i}(K)=\mathrm{W}_{i}\left(K ; B^{3}\right)$. Thus we get that (5.5) is a strengthening of Osserman's inequality.

Remark 5.2. In [15, p. 175] it is shown that for any convex body $K \in \mathcal{K}_{0}^{n}$ with inradius $\mathrm{r}=\mathrm{r}(K)$ and for all $-\mathrm{r} \leq \lambda \leq 0$

$$
\mathrm{V}\left(K_{\lambda}\right) \geq \mathrm{V}(K)-3|\lambda| \mathrm{W}_{1}(K)+2 \lambda^{2} \mathrm{~W}_{2}(K)+\lambda^{2} \mathrm{~W}_{2}\left(K_{\lambda}\right),
$$

where equality holds for all $-\mathrm{r} \leq \lambda \leq 0$ if and only if $K$ is a 1-tangential body of $K_{-\mathrm{r}}+\mathrm{r} B^{3}$.

We would like to point out that the characterization of the equality case is not correct since, in fact, if equality holds in (5.6) then $K$ is a 1-tangential body of $K_{-\mathrm{r}}+\mathrm{r} B^{3}$, but not every 1-tangential body of $K_{-\mathrm{r}}+\mathrm{r} B^{3}$ satisfies (5.6); condition (2.3) is needed (see Figure 2). It has however no influence in the characterization of the equality case in the interesting inequality

$$
\mathrm{V}(K)-3 \mathrm{r}(K) \mathrm{W}_{1}(K)+2 \mathrm{r}(K)^{2} \mathrm{~W}_{2}(K) \leq 0,
$$

which is obtained in [15, p. 177] as a consequence of (5.6): equality holds if and only if $K$ is a 1-tangential body of the ball, which is true since in this case the kernel $K_{-\mathrm{r}}$ is just a point, and hence condition (2.3) holds trivially.

Inequality in Corollary 5.1 for $n=3$ and $E=B^{3}$,

$$
\mathrm{V}\left(K_{\lambda}\right) \geq \mathrm{V}(K)-3|\lambda| \mathrm{W}_{1}(K)+3 \frac{\lambda^{2}}{2} \mathrm{~V}\left(K, K^{*}, E\right)-3 \int_{\lambda}^{0} t \mathrm{~V}\left(K_{t}, K^{*}, E\right) d t,
$$

strengthens (5.6). The proof of this fact is analogous to the one in [3, p. 3982].

Acknowledgements. The authors would like to thank the anonymous referee for valuable comments and suggestions. 


\section{REFERENCES}

[1] G. Bol, Beweis einer Vermutung von H. Minkowski, Abh. Math. Sem. Univ. Hamburg 15 (1943), 37-56.

[2] T. Bonnesen and W. Fenchel, Theorie der konvexen Körper. Springer, Berlin, 1934, 1974. English translation: Theory of convex bodies. Edited by L. Boron, C. Christenson and B. Smith. BCS Associates, Moscow, ID, 1987.

[3] N. S. Brannen, The Wills conjecture, Trans. Amer. Math. Soc. 349 (10) (1997), 3977-3987.

[4] A. Dinghas, Bemerkung zu einer Verschärfung der isoperimetrischen Ungleichung durch H. Hadwiger, Math. Nachr. 1 (1948), 284-286.

[5] M. Fradelizi, A. Giannopoulos, M. Meyer, Some inequalities about mixed volumes, Israel J. Math. 135 (2003), 157-179.

[6] P. M. Gruber, Convex and Discrete Geometry. Springer, Berlin Heidelberg, 2007.

[7] H. Hadwiger, Altes und Neues über konvexe Körper. Birkhäuser Verlag, Basel und Stuttgart, 1955.

[8] H. Hadwiger, Vorlesungen über Inhalt, Oberfläche und Isoperimetrie. Springer-Verlag, Berlin-Göttingen-Heidelberg, 1957.

[9] M. Henk, M. A. Hernández Cifre, Notes on the roots of Steiner polynomials, Rev. Mat. Iberoamericana 24 (2) (2008), 631-644.

[10] M. A. Hernández Cifre, E. Saorín, On differentiability of quermassintegrals, to appear in Forum Math.

[11] M. A. Hernández Cifre, E. Saorín, On the volume of inner parallel bodies, to appear in Adv. Geom.

[12] G. Matheron, La formule de Steiner pour les érosions, J. Appl. Prob. 15 (1978), 126-135.

[13] R. Osserman, Bonnesen-style isoperimetric inequalities, Amer. Math. Monthly 86 (1) (1979), 1-29.

[14] J. R. Sangwine-Yager, Inner Parallel Bodies and Geometric Inequalities. Ph.D. Thesis Dissertation, University of California Davis, 1978.

[15] J. R. Sangwine-Yager, A Bonnesen-style inradius inequality in 3-space, Pacific J. Math. 134 (1) (1988), 173-178.

[16] R. Schneider, On the Aleksandrov-Fenchel inequality, In Discrete Geometry and Convexity (eds. J. E. Goodman, E. Lutwak, J. Malkevitch \& R. Pollack), Ann. New York Acad. Sci. 440 (1985), 132-141.

[17] R. Schneider, Convex Bodies: The Brunn-Minkowski Theory, Cambridge University Press, Cambridge, 1993.

Departamento de Matemáticas, Universidad de Murcia, Campus de EspinarDO, 30100-Murcia, SPAin

E-mail address: mhcifre@um.es

Departamento de Matemáticas, Universidad de Murcia, Campus de EspinarDO, 30100-MurCIA, SPAIN

E-mail address: esaorin@um.es 